\title{
Rare Kaon Decays Beyond the Standard Model
}

\author{
Cecilia Tarantino ${ }^{* \dagger}$ \\ Physik Department, Technische Universität München, D-85748 Garching, Germany \\ E-mail: tarantinodfis.uniroma3.it
}

\begin{abstract}
The rare kaon decays $K_{L} \rightarrow \pi^{0} v \bar{v}, K^{+} \rightarrow \pi^{+} v \bar{v}, K_{L} \rightarrow \pi^{0} e^{+} e^{-}$and $K_{L} \rightarrow \pi^{0} \mu^{+} \mu^{-}$are theoretically very clean and, being strongly CKM suppressed, highly sensitive to New Physics (NP). Recent Flavour Physics analyses show that they represent unique probes for revealing NP effects and to provide information on the NP flavour structure. After a brief discussion of the main properties that make rare $K$ decays so promising and of the basic ideas of the most interesting NP models, we review the results of recent phenomenological analyses both within and beyond the framework of Minimal Flavour Violation (MFV), where the sources of flavour violation are the same as in the Standard Model. Within MFV we present the expectations found for rare $K$ decays from a model-independent analysis and in three MFV models: the Littlest Higgs (LH) model, the (extra-dimension) Appelquist-Cheng-Dobrescu model and the Minimal Supersymmetric Standard Model (MSSM) with MFV. Beyond MFV, we discuss the results recently found within the MSSM (without MFV), the LH model with T-parity (LHT) and the 3-3-1 ( $\left.Z^{\prime}\right)$ model. While in MFV models only small $(<30 \%)$ NP effects are allowed in the branching ratios of $K_{L} \rightarrow \pi^{0} v \bar{v}$, $K^{+} \rightarrow \pi^{+} v \bar{v}, K_{L} \rightarrow \pi^{0} e^{+} e^{-}$and $K_{L} \rightarrow \pi^{0} \mu^{+} \mu^{-}$, beyond MFV, in particular in the MSSM and in the LHT model, large (up to an order of magnitude) enhancements w.r.t. the SM turn out to be possible.
\end{abstract}

\section{KAON International Conference}

May 21-25 2007

Laboratori Nazionali di Frascati dell'INFN, Rome, Italy

\footnotetext{
* Speaker.

$\dagger$ It is a pleasure to thank the organizers of "Kaon'07" for the very interesting conference realized at the Laboratori Nazionali di Frascati. I am also grateful to Andrzej J. Buras and Federico Mescia for inspiring some important issues of my talk and to Ulrich Haisch for a careful reading of the manuscript. This work is partially supported by the Cluster of Excellence 'Origin and Structure of the Universe' and by the German 'Bundesministerium für Bildung und Forschung' under contract 05HT6WOA.
} 


\section{Rare Kaon Decays as Unique Probes of New Physics}

The rare kaon decays $K_{L} \rightarrow \pi^{0} v \bar{v}, K^{+} \rightarrow \pi^{+} v \bar{v}, K_{L} \rightarrow \pi^{0} e^{+} e^{-}$and $K_{L} \rightarrow \pi^{0} \mu^{+} \mu^{-}$play a fundamental role in looking for New Physics (NP) beyond the Standard Model (SM), because they are theoretically very clean and highly sensitive to NP, being strongly suppressed within the SM. ${ }^{1}$ Moreover, their theoretical cleanness is not due to any cancellation and remains true also beyond the SM, thus allowing a precise investigation of NP effects.

The decays $K \rightarrow \pi v \bar{v}$ are short-distance dominated and involve matrix elements that can be accurately extracted from the experimental data on $K_{\ell 3}$ decays $[2,3]$, such that the intrinsic theoretical uncertainty is smaller than $5 \%$. In $K_{L} \rightarrow \pi^{0} v \bar{v}$, as $K_{L} \simeq\left(K^{0}+\bar{K}^{0}\right) / \sqrt{2}$ and the $K^{0} \rightarrow \pi^{0}$ and $\bar{K}^{0} \rightarrow \pi^{0}$ matrix elements differ by a minus sign, the CKM contributions involved are the CP-violating imaginary parts $\operatorname{Im}\left(\lambda_{i}\right) \equiv \operatorname{Im}\left(V_{i d} V_{i s}^{*}\right)(i=u, c, t)$. Through the GIM mechanism $\operatorname{Im}\left(\lambda_{u}\right)$ can be expressed in terms of $\operatorname{Im}\left(\lambda_{t}\right)$ and $\operatorname{Im}\left(\lambda_{c}\right)$, both strongly Cabibbo suppressed $\left(\operatorname{Im}\left(\lambda_{t}\right) \sim \operatorname{Im}\left(\lambda_{c}\right) \sim \mathscr{O}\left(\lambda^{5}\right)\right)$. Moreover, the short-distance loop-function $X$ describing $K_{L} \rightarrow \pi^{0} v \bar{v}$ is highly suppressed if a small quark mass runs in the loop, thus making the charm contribution negligible. It turns out, then, that $K_{L} \rightarrow \pi^{0} v \bar{v}$ is CKM suppressed as $\mathscr{O}\left(\lambda^{5}\right)$ and fully dominated by the top contribution. The decay $K^{+} \rightarrow \pi^{+} v \bar{v}$ is also described by the short-distance function $X$ but, in the $K^{+}$decay, both imaginary and real parts of the CKM contributions are involved and, being $\operatorname{Re}\left(\lambda_{c}\right) \sim O(\lambda) \gg \operatorname{Re}\left(\lambda_{t}\right) \sim O\left(\lambda^{5}\right)$, the charm contribution cannot be neglected in this case. Its perturbative calculation requires some care as the charm scale $\left(m_{c} \sim 1 \mathrm{GeV}\right)$ is not as high as the top one $\left(m_{t} \sim 10^{2} \mathrm{GeV}\right)$. Recently, however, the uncertainty on the charm contribution has been significantly reduced thanks to the computation of the NNLO corrections [4] and the study of longdistance and dimension- 8 operator effects [5]. This improvement, together with the determination [3] of the operator matrix elements for both $K \rightarrow \pi v \bar{v}$ and $K_{L} \rightarrow \pi^{0} \ell^{+} \ell^{-}$from $K_{\ell 3}$ data using chiral perturbation theory beyond $\mathrm{LO}$, represents the main theoretical progress in rare $K$ decays.

The decays $K_{L} \rightarrow \pi^{0} \ell^{+} \ell^{-}(\ell=e, \mu)$, though not as theoretically clean as the golden modes $K \rightarrow \pi v \bar{v}$, have an intrinsic theoretical uncertainty smaller than $10 \%$ and represent very promising channels for their peculiar sensitivity to NP. They involve three contributions of comparable size: a direct CP-violating contribution of short-distance origin, an indirect $\mathrm{CP}$-violating contribution that can be determined from the experimental measurement of $K_{S} \rightarrow \pi^{0} \ell^{+} \ell^{-}$and a long-distance CPconserving contribution that can be determined from the experimental data on $K_{L} \rightarrow \pi^{0} \gamma \gamma$. This last contribution comes from the two-photon channel $K_{L} \rightarrow \pi^{0}(\gamma \gamma)_{J=0,2} \rightarrow \pi^{0}\left(\ell^{+} \ell^{-}\right)_{J=0,2}$ that, being of long-distance origin, represents the main source of uncertainty, now under control thanks to recent studies [6, 7]. Moreover, the two-photon contribution distinguishes between the muon and electron modes as the $J^{\mathrm{CP}}=0^{++}$channel is helicity suppressed and, therefore, significant only in the (heavier) muon case. More in general, a relevant peculiarity of the $K_{L} \rightarrow \pi^{0} \ell^{+} \ell^{-}$decays is that the different impact of helicity suppressed contributions to the muon and electron modes makes the comparison of $K_{L} \rightarrow \pi^{0} \mu^{+} \mu^{-}$and $K_{L} \rightarrow \pi^{0} e^{+} e^{-}$a powerful tool to reveal the properties of NP [8].

Finally, we collect in table 1 the SM theoretical predictions for the four rare kaon decays in question compared to present experimental measurements or limits, to underline the accuracy of the SM predictions and the large room for NP still allowed by the experiments.

\footnotetext{
${ }^{1}$ For an extensive discussion of the SM theoretical predictions of rare kaon decays and for a complete list of references we address the reader to ref. [1].
} 


\begin{tabular}{||c||c|c||}
\hline Decay & SM & Experiment \\
\hline$K^{+} \rightarrow \pi^{+} v \bar{v}$ & $(7.8 \pm 0.8) \cdot 10^{-11}[3]$ & $\left(14.7_{-8.9}^{+13}\right) \cdot 10^{-11}[9]$ \\
$K_{L} \rightarrow \pi^{0} v \bar{v}$ & $(2.5 \pm 0.4) \cdot 10^{-11}[3]$ & $<2.1 \cdot 10^{-7}[10]$ \\
$K_{L} \rightarrow \pi^{0} e^{+} e^{-}$ & $\left(3.5_{-0.9}^{+1.0}\right) \cdot 10^{-11}[8]$ & $<2.8 \cdot 10^{-10}[11]$ \\
$K_{L} \rightarrow \pi^{0} \mu^{+} \mu^{-}$ & $(1.4 \pm 0.3) \cdot 10^{-11}[8]$ & $<3.8 \cdot 10^{-10}[12]$ \\
\hline
\end{tabular}

Table 1: SM predictions and experimental measurements of the four rare kaon decays

\section{The Most Interesting NP Models}

\subsection{Distinction between Models with and without Minimal Flavour Violation}

In this section we discuss the basic features of the most appealing NP models, i.e. the NP models that solve at least some of the open questions of the SM (first of all the so-called little hierarchy problem), remaining simple enough to be predictive and compatible with electroweak (ew) precision tests and with flavour constraints. We recall that the little hierarchy problem occurs as the NP scale is required to be low $\left(\Lambda_{\mathrm{NP}} \sim 1 \mathrm{TeV}\right)$ to explain the lightness of the Higgs mass without fine-tuning, while it is constrained to be quite high $\left(\Lambda_{\mathrm{NP}} \sim 5-10 \mathrm{TeV}\right)$ by phenomenological analyses where NP effects are taken into account by higher dimensional operators [13, 14].

NP models can satisfy or not the Minimal Flavour Violation (MFV) hypothesis, consisting in the absence of new sources of flavour violation in addition to the SM. MFV provides a natural, though not very exciting, explanation to the great success of the SM in Flavour Physics. There are two different formulations of MFV: a pragmatic and a theoretical one. The pragmatic formulation [15] states that a model is a MFV model if it satisfies two constraints: the only source of flavour violation is the CKM matrix (as in the SM) and it involves only the SM operators. Because of this second property, the pragmatic formulation is also called Constrained MFV (CFMV) [16]. The theoretical formulation [17] consists in building a MFV model by using as flavour violating building blocks only the SM Yukawa couplings. Both formulations are valid, similar indeed, with a slight difference due to the absence of new operators in the CMFV case. Finally, NP models going beyond MFV are those that introduce new sources of flavour violation in addition to the SM.

In order to see how NP effects can appear in Flavour Physics observables, let us consider a generic weak amplitude $A$ that can be schematically written as [18]

$$
A=V_{\mathrm{CKM}}^{i}\left\{B_{i} \eta_{\mathrm{QCD}}^{i}\left[F_{\mathrm{SM}}^{i}+F_{\mathrm{CMFV}}^{i}\right]+B_{i}^{\mathrm{MFV}}\left(\eta_{\mathrm{QCD}}^{i}\right)^{\mathrm{MFV}} F_{\mathrm{MFV}}^{i}\right\}+V_{\mathrm{NEW}}^{i} B_{i}^{\mathrm{NEW}}\left(\eta_{\mathrm{QCD}}^{i}\right)^{\mathrm{NEW}} F_{\mathrm{NEW}}^{i},
$$

where $F^{i}$ are the (perturbative) short-distance functions ${ }^{2}, \eta^{i}$ are their NLO corrections in QCD, $B_{i}$ are the (non-perturbative) low energy parameters describing the operator matrix elements and $V_{\text {CKM }}^{i}$ denote the CKM combinations entering the decay. Within CMFV the only NP effect is a modification of the short-distance functions $F_{i}$, while in MFV new operators and therefore new $B$-parameters can also appear. A more general NP model going beyond MFV, in addition, can introduce new sources of flavour violation different from the CKM ones, denoted by $V_{\mathrm{NEW}}^{i}$.

\footnotetext{
${ }^{2}$ The short-distance function involved in $K_{L} \rightarrow \pi^{0} v \bar{v}$ and $K^{+} \rightarrow \pi^{+} v \bar{v}$ is the loop-function $X$ calculated from Zpenguin and box diagrams. In $K_{L} \rightarrow \pi^{0} \ell^{+} \ell^{-}$, in addition to $X$, the loop-functions $Y$ and $Z$ are also involved, which require the calculation of the photon-penguin diagram.
} 


\subsection{MFV Models}

Very interesting NP models that satisfy MFV are the Littlest Higgs (LH) model [19], the Appelquist-Cheng-Dobrescu (ACD) model [20] and the Minimal Supersymmetric Standard Model (MSSM) with MFV [21].

The LH model [19] solves the little hierarchy problem, by interpreting the Higgs boson as a pseudo-Goldstone boson of a global symmetry, that being massless at tree-level can be naturally kept light. The global symmetry in question is $S U(5)$, spontaneously broken to $S O(5)$ at a NP scale $f \sim \mathscr{O}(\mathrm{TeV})$. The SM problematic quadratic corrections to the Higgs mass are cancelled in the LH model by the contributions of new particles having the same spin-statistics of the SM ones: heavy gauge bosons $\left(W_{H}^{ \pm}, Z_{H}, A_{H}\right)$, the heavy top $(T)$ and a scalar triplet $(\Phi)$. These new particles, however, introduce tree-level corrections such that the NP scale is constrained to be quite large $(f>2-3 \mathrm{TeV})$ by ew precision tests and the model becomes to be less appealing.

The ACD model [20] is characterized by one universal extra-dimension, that means that the SM particles propagate in 5 dimensions. In 4 dimensions the extra-dimension is taken into account by the appearance of new (heavy) Kaluza-Klein (KK) modes. The model satisfies a discrete parity called KK-parity that, implying an even number of KK modes in vertices, avoids undesired treelevel corrections. A very nice feature of this model is its simplicity. Typically, in fact, flavour observables can be expressed at LO in terms of only one parameter: the compactification radius $R$.

The MSSM [21] is the supersymmetric extension of the SM with minimal matter content. The new particles are the so-called sparticles, i.e. superpartners of the SM ones with opposite spinstatistics and heavy masses (that cancel the SM quadratic corrections to Higgs mass), and two Higgs doublets $H_{u}$ and $H_{d}$ whose vacuum expectation values define the model parameter $\tan \beta \equiv v_{u} / v_{d}$. The R-parity, i.e. the discrete symmetry inducing an even number of sparticles in vertices, is introduced in order to avoid undesired tree-level contributions, e.g. to the proton decay. The MSSM can satisfy MFV once the soft breaking terms are imposed to be adequate combinations of Yukawa couplings. Moreover, for small values of $\tan \beta$ the effects of new operators are unimportant and the model satisfies CMFV (unless the MSSM parameter $\mu$ is large, $\mu>500$ [22]). At large $\tan \beta$, instead, the impact of new operators generated by Higgs contributions becomes important.

\subsection{Non-MFV Models}

A very interesting NP model going beyond MFV is certainly the MSSM [21] where the soft breaking terms are left free to introduce new sources of flavour violation. In particular, 27 new flavour changing couplings appear in the squark propagators with significant effects in flavour observables.

An interesting alternative to the MSSM is the LH model when a discrete symmetry, T-parity [23], is introduced. T-parity acts similarly to R-parity in supersymmetry or KK-parity in extradimension models, assigning opposite parities to SM and new particles and, thus, forbidding dangerous tree-level contributions. The LH model with T-parity (LHT) satisfies ew precision tests already at a quite low NP scale $(f>500 \mathrm{GeV})$, as required to solve the little hierarchy problem. Once T-parity is introduced, new particles appear in addition to the heavy gauge bosons, the heavy top and the scalar triplet already present without T-parity. They are the so-called mirror fermions, i.e. (same spin-statistics) partners of the SM fermions, characterized by new flavour interactions with important effects in flavour observables. 
Another quite simple and predictive extension of the SM is represented by the 3-3-1 model [24], whose name comes from its extended gauge group $S U(3)_{c} \times S U(3)_{L} \times U(1)_{X}$. The extension of the left-handed gauge group to $S U(3)_{L}$ requires the introduction of new particles: heavy gauge bosons and heavy fermions to complete fermion multiplets. Different fermion generations can belong to different representations of $S U(3)_{L}$ and, indeed, the first two quark generations are set in triplets, while the third quark generation in an antitriplet. This choice is welcome as it explains the necessity of three fermion generations in order to obtain anomaly cancellation and QCD asymptotic freedom. A consequence of the peculiarity of the third generation is that the neutral heavy gauge boson $Z^{\prime}$ (the model is also called $Z^{\prime}$-model) can transmit flavour changing neutral currents at treelevel, with important effects in flavour observables.

\section{Expectations for Rare Kaon Decays Beyond the SM}

\subsection{Within MFV}

A model independent study of Flavour Physics within CMFV has been performed in [25], taking into account the available information from the Unitarity Triangle (UT) analysis and from the measurements of $\operatorname{Br}\left(B \rightarrow X_{s} \gamma\right)$ and $\operatorname{Br}\left(B \rightarrow X_{s} \ell^{+} \ell^{-}\right)$. The goal of that study was the determination of the range allowed for the short-distance function $C$ (i.e. the Z-penguin contribution, while NP effects in box and gluon-penguin diagrams, typically small in MFV models, were neglected) and the evaluation of upper bounds for rare decays. It was found that the enhancements can be at most of $30 \%$ for $\operatorname{Br}\left(K_{L} \rightarrow \pi^{0} v \bar{v}\right)$ and $\operatorname{Br}\left(K^{+} \rightarrow \pi^{+} v \bar{v}\right)$, whose accurate experimental measurements are certainly looked forward in view of their high sensitivity to NP. The analysis of [25] has just been updated and improved in [26] by including the constraints from the observables related to the $Z \bar{b} b$ vertex. The main effect of these new constraints is the exclusion of the negative (non-SM) sign solution for the $C$ function. As a consequence, $\operatorname{Br}\left(K_{L} \rightarrow \pi^{0} v \bar{v}\right)$ and $\operatorname{Br}\left(K^{+} \rightarrow \pi^{+} v \bar{v}\right)$ turn out to be bounded also from below and to lie in the following 95\% C.L. ranges [26]

$$
\operatorname{Br}\left(K^{+} \rightarrow \pi^{+} v \bar{v}\right)_{\mathrm{CMFV}} \in[4.3,10.7] \cdot 10^{-11}, \quad \operatorname{Br}\left(K_{L} \rightarrow \pi^{0} v \bar{v}\right)_{\mathrm{CMFV}} \in[1.6,4.4] \cdot 10^{-11}
$$

Furthermore, in [25] a strong correlation between $\operatorname{Br}\left(K_{L} \rightarrow \pi^{0} v \bar{v}\right)$ and $\operatorname{Br}\left(K^{+} \rightarrow \pi^{+} v \bar{v}\right)$ has been pointed out. This is expected from the MFV relation [17, 27]

$$
\left.\frac{B r\left(K_{L} \rightarrow \pi^{0} v \bar{v}\right)}{B r\left(K^{+} \rightarrow \pi^{+} v \bar{v}\right)}\right|_{\mathrm{MFV}}=\left.\frac{B r\left(K_{L} \rightarrow \pi^{0} v \bar{v}\right)}{B r\left(K^{+} \rightarrow \pi^{+} v \bar{v}\right)}\right|_{\mathrm{SM}} \cdot\left[1+\varepsilon_{c} \operatorname{sign}(X)\right],
$$

where two branches are distinguished by the sign of the $X$ function and $\varepsilon_{c}$ denotes a small charm contribution coming from $K^{+} \rightarrow \pi^{+} v \bar{v}$. Within CMFV, the exclusion of the negative sign solution for $C[26]^{3}$ leaves only one branch in the correlation (3.2).

For completeness we mention the results found from the analyses of rare kaon decays in specific CMFV models. In the LH model, where the NP scale is constrained to be quite large $(f>2-3 \mathrm{TeV})$ by ew precision tests, NP effects in flavour observables turn out to be suppressed and, in particular, in $\operatorname{Br}\left(K_{L} \rightarrow \pi^{0} v \bar{v}\right)$ and $\operatorname{Br}\left(K^{+} \rightarrow \pi^{+} v \bar{v}\right)$ can amount at most to $15 \%$ [28]. Similar enhancements turn out to be allowed in the ACD model for values of the compactification radius

\footnotetext{
${ }^{3}$ With the assumption of $[25,26]$ that no NP effects appear in box diagrams, the NP contributions to the $X$ function coincide with those to the (Z-penguin) $C$ function and, therefore, $\operatorname{sign}(X) \equiv \operatorname{sign}(C)$.
} 
$1 / R \simeq 200 \mathrm{GeV}$ [29]. Even smaller enhancements $(<5 \%)$ are found [31] if $1 / R>500-600 \mathrm{GeV}$, as required by the $B r\left(B \rightarrow X_{s} \gamma\right)$ constraint after including the recent NNLO corrections [30]. In the MSSM with MFV and small $(<30) \tan \beta$, the NP effects to $\operatorname{Br}\left(K_{L} \rightarrow \pi^{0} v \bar{v}\right)$ and $B r\left(K^{+} \rightarrow \pi^{+} v \bar{v}\right)$ turn out to be naturally small $(\sim 10 \%)$ [32], but could saturate the model-independent bounds of $[25,26]$. At large $\tan \beta$, charged Higgs loops generate a new operator $\bar{s} \gamma^{\mu}\left(1+\gamma_{5}\right) d \bar{v} \gamma^{\mu}\left(1-\gamma_{5}\right) v$, not suppressed by any light quark mass. Nevertheless, it has been pointed out [33] that within MFV its contribution doesn't modify significantly the small $\tan \beta$ results just mentioned above.

Concerning the decays $K_{L} \rightarrow \pi^{0} \ell^{+} \ell^{-}$, in models without new operators the NP effects in $K_{L} \rightarrow$ $\pi^{0} \ell^{+} \ell^{-}$are generally smaller than in $K \rightarrow \pi v \bar{v}$. Within CMFV the enhancements of $\operatorname{Br}\left(K_{L} \rightarrow\right.$ $\left.\pi^{0} \ell^{+} \ell^{-}\right)$are expected to be utmost of $10 \%$. Still, a combined measurement of electron and muon modes would be very interesting to reveal helicity suppressed NP effects.

Finally, we emphasize that the absence, within CMFV, of large departures from the SM in rare kaon decays, though not very exciting, provides a strong message: if a large enhancement was found in rare kaon decays then all CMFV models would become very unlikely.

\subsection{Beyond MFV}

We discuss now the expectations for rare kaon decays beyond MFV in the three NP models briefly described in Section 2.3: the MSSM [21] with a general flavour structure, the LHT model [23] and the 3-3-1 ( $\left.Z^{\prime}\right)$ model [24].

Various analyses of rare kaon decays have been performed [32]-[38] in the MSSM with a general flavour structure. ${ }^{4}$ In order to obtain simple analytic expressions for $s-d$ transitions, involved in rare $\mathrm{K}$ decays, it is useful to work in a basis where the gauge interactions of the SM down quarks with gauginos and neutralinos are flavour diagonal. In this basis the squark mass matrices, made up of four blocks corresponding to the squark chiralities $L L, L R, R L$ and $R R$, are non-diagonal in flavour space. It is convenient to use the so-called Mass Insertion Approximation (MIA) to expand them in the small off-diagonal entries $\delta$ 's. In rare kaon decays the dominant NP effects are represented by chargino up-squark loops, with double insertions of $\delta_{L R}^{U}$ not suppressed by $\mathscr{O}\left(M_{W} / M_{S U S Y}\right)$ nor CKM, and by charged-Higgs top-quark loops, with $\delta_{R R}^{D}$ contributions not suppressed by any light quark mass and enhanced by $\tan ^{4} \beta$.

In [32] it has been found that the mass insertions $\delta_{L R}^{U}$ are essentially not constrained by present measurements of $B$ and $K$ observables, as shown in fig. 1. From fig. 1 it is also evident that rare kaon decays, e.g. $\operatorname{Br}\left(K^{+} \rightarrow \pi^{+} v \bar{v}\right)$, are potentially the most constraining for the $\delta_{L R}^{U}$ 's. In [33], then, it has been found that precise measurements of $\operatorname{Br}\left(K_{L} \rightarrow \pi^{0} v \bar{v}\right)$ and $\operatorname{Br}\left(K^{+} \rightarrow \pi^{+} v \bar{v}\right)$ could also provide the most stringent bounds on the mass insertions $\delta_{R R}^{D}$. Furthermore, the analysis of [32], where $\tan \beta<30$, finds that $\mathscr{O}(1)$ enhancements $\operatorname{Br}\left(K_{L} \rightarrow \pi^{0} v \bar{v}\right)$ and $\operatorname{Br}\left(K^{+} \rightarrow \pi^{+} v \bar{v}\right)$ are possible. From a very general scan [37] over the MSSM parameters, even enhancements of one order of magnitude turn out to be possible. Concerning the decays $K_{L} \rightarrow \pi^{0} \ell^{+} \ell^{-}$, at large $\tan \beta$ the contributions of the (pseudo)scalar operators $\bar{s} d \bar{\ell} \ell$ and $\bar{s} d \bar{\ell} \gamma_{5} \ell$ are enhanced. As they are helicity suppressed, they affect the muon mode only and could provide a clear signal.

We now discuss the results found in ref. [40] for rare kaon decays within the LHT model. As discussed in Section 2.3, after introducing T-parity the model allows for new sources of flavour

\footnotetext{
${ }^{4}$ For an extensive discussion and bibliography on rare kaon decays in the MSSM we address the reader to ref. [39].
} 


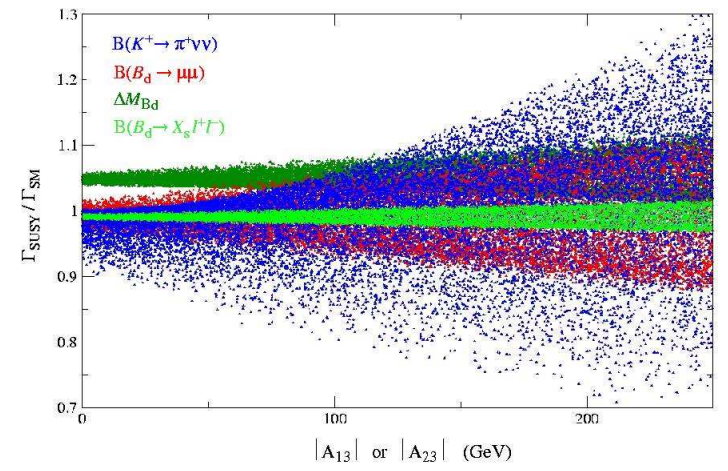

Figure 1: Dependence, within the MSSM, of flavour observables on the trilinear couplings $A_{i 3}(i=1,2)$ or, equivalently, on the $\delta_{L R}^{U}$ mass insertions as with a good approximation $\left(\delta_{L R}^{U}\right)_{i 3} \propto m_{t} A_{i 3}(i=1,2)$ [32].

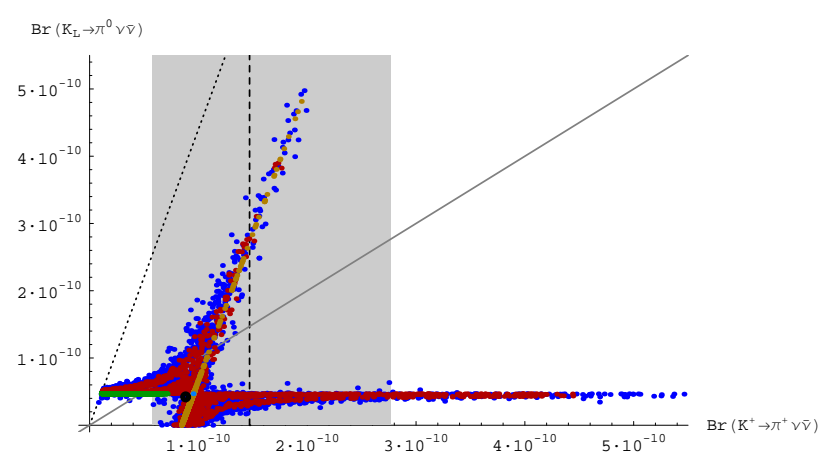

Figure 2: $\operatorname{Br}\left(K_{L} \rightarrow \pi^{0} v \bar{v}\right)$ as a function of $\operatorname{Br}\left(K^{+} \rightarrow \pi^{+} v \bar{v}\right)$ in the LHT model [40]. The shaded area represents the experimental $1 \sigma$-range for $\mathrm{Br}\left(K^{+} \rightarrow \pi^{+} v \bar{v}\right)$. The model-independent Grossman-Nir bound [42] is displayed by the dotted line, while the solid line separates the two areas where $\operatorname{Br}\left(K_{L} \rightarrow \pi^{0} v \bar{v}\right)>$ $(<) \operatorname{Br}\left(K^{+} \rightarrow \pi^{+} v \bar{v}\right)$.

violation induced by mirror fermions. In particular, a new mixing matrix $V_{H d}$ that governs the interactions of a SM quark with a mirror quark and a heavy gauge boson is involved in the quark sector. It is parameterized in terms of three new mixing angles and three new (CP-violating) phases [41] and its hierarchy can be completely different from the CKM. As a consequence, the strong CKM suppression of rare kaon decays present in the SM can have no analogy in the mirror sector and large NP effects may occur. Indeed, this is the main result found in [40] and shown in fig. 2, where two branches in the $\operatorname{Br}\left(K^{+} \rightarrow \pi^{+} v \bar{v}\right)-B r\left(K_{L} \rightarrow \pi^{0} v \bar{v}\right)$ plane result from a general scan over the LHT parameters. They are selected by the indirect CP-violation parameter $\varepsilon_{K}$, which turns out to be the most stringent constraint. The horizontal branch corresponds to an enhancement of $\operatorname{Br}\left(K^{+} \rightarrow \pi^{+} v \bar{v}\right)$ up to a factor 5, with $\operatorname{Br}\left(K_{L} \rightarrow \pi^{0} v \bar{v}\right)$ close to the SM prediction. The oblique branch, instead, signals that an enhancement of $B r\left(K_{L} \rightarrow \pi^{0} v \bar{v}\right)$ up to a factor 10 is possible, with a simultaneous enhancement of $\operatorname{Br}\left(K^{+} \rightarrow \pi^{+} v \bar{v}\right)$ of at most a factor 3. In [40] the decays $K_{L} \rightarrow$ $\pi^{0} \ell^{+} \ell^{-}$have also been studied, finding enhancements up to a factor 2 w.r.t. the SM and a strong correlation between the muon and the electron modes and with $K_{L} \rightarrow \pi^{0} v \bar{v}$. Another important result of ref. [40] concerns the UT angle $\beta$, for which a difference between the value obtained from $B_{d} \rightarrow J_{\psi} K_{s}$ and a future determination from $K_{L} \rightarrow \pi^{0} v \bar{v}$ would be a clear signal of NP. Within the LHT model the universality between $B$ and $K$ systems is strongly violated and such a signal could be easily seen. More recently, the potentiality of the direct CP-violating parameter $\varepsilon^{\prime} / \varepsilon$ in 
constraining rare kaon decays within the LHT model has been studied [43]. While the experimental measurement of $\varepsilon^{\prime} / \varepsilon$ is accurate at the $10 \%$ level $\left(\left(\varepsilon^{\prime} / \varepsilon\right)_{\text {exp. }}=(17 \pm 2) \cdot 10^{-4}[44]\right)$, the theoretical prediction requires to know the value for the gluon penguin matrix element which still has large (non-perturbative) uncertainties. A significant theoretical progress would be definitely welcome, as $\varepsilon^{\prime} / \varepsilon$ has been found to be potentially very constraining for the LHT parameters and in particular for rare kaon decays. With an accurate theoretical prediction at hand, $\varepsilon^{\prime} / \varepsilon$ would become a strong constraint also for the MSSM, as pointed out in [38, 45].

We finally discuss the results found in [46] for rare kaon decays within the 3-3-1 $\left(Z^{\prime}\right)$ model. As discussed in Section 2.3, in this model significant NP effects are expected in Flavour Physics as the heavy gauge boson $Z^{\prime}$ can transmit flavour changing neutral currents at tree-level. We note that these tree-level effects are due to the peculiar role of the third quark generation, set in a different $S U(3)_{L}$ representation w.r.t. the first two. They are, instead, absent in ew observables that turn out to induce only mild constraints on the NP scale. Significant NP effects are found in $K_{L} \rightarrow \pi^{0} v \bar{v}$ and $K^{+} \rightarrow \pi^{+} v \bar{v}$ showing two possible branches in the $\operatorname{Br}\left(K^{+} \rightarrow \pi^{+} v \bar{v}\right)-B r\left(K_{L} \rightarrow \pi^{0} v \bar{v}\right)$ plane, with a pattern similar to the LHT one (see fig. 2). Here, however, smaller enhancements are allowed (up to a factor 4 and 2 for $\operatorname{Br}\left(K_{L} \rightarrow \pi^{0} v \bar{v}\right)$ and $\operatorname{Br}\left(K^{+} \rightarrow \pi^{+} v \bar{v}\right)$, respectively), because of the leptophobic nature of the $Z^{\prime} \bar{\ell} \ell$ coupling, suppressed by the weak mixing angle as $\sqrt{1-\sin ^{2} \theta_{W}}$. Concerning $K_{L} \rightarrow \pi^{0} \ell^{+} \ell^{-}$, enhancements up to a factor 3 w.r.t. the SM result to be possible and strong correlations are found between the electron and muon modes and with $K_{L} \rightarrow \pi^{0} v \bar{v}$.

In conclusion, we point out that while within MFV only small $(<30 \%) \mathrm{NP}$ effects can be expected in $K_{L} \rightarrow \pi^{0} v \bar{v}, K^{+} \rightarrow \pi^{+} v \bar{v}$ and $K_{L} \rightarrow \pi^{0} \ell^{+} \ell^{-}$, much larger (up to an order of magnitude) effects are allowed in non-MFV models, in particular in the MSSM and in the LHT model. Rare kaon decays clearly represent powerful tools to look for NP and to investigate its flavour structure.

\section{References}

[1] U. Haisch, these proceedings. A. J. Buras, F. Schwab and S. Uhlig, hep-ph/0405132.

[2] W. J. Marciano and Z. Parsa, Phys. Rev. D 53 (1996) 1.

[3] F. Mescia and C. Smith, 0705.2025 [hep-ph].

[4] A. J. Buras et al., Phys. Rev. Lett. 95 (2005) 261805 hep-ph/0508165. A. J. Buras et al., JHEP 0611 (2006) 002 hep-ph/0603079.

[5] G. Isidori, F. Mescia and C. Smith, Nucl. Phys. B 718 (2005) 319 hep-ph/0503107. A. F. Falk, A. Lewandowski and A. A. Petrov, Phys. Lett. B 505 (2001) 107 hep-ph/0012099.

[6] G. Buchalla, G. D’Ambrosio and G. Isidori, Nucl. Phys. B 672 (2003) 387 hep-ph/0308008.

[7] G. Isidori, C. Smith and R. Unterdorfer, Eur. Phys. J. C 36 (2004) 57 hep-ph/0404127.

[8] F. Mescia, C. Smith and S. Trine, JHEP 0608 (2006) 088 hep-ph/0606081.

[9] S. Adler et al. [E787 Collaboration], Phys. Rev. Lett. 88 (2002) 041803 hep-ex/0111091. V. V. Anisimovsky et al. [E949 Collaboration], Phys. Rev. Lett. 93 (2004) 031801 hep-ex/0403036.

[10] J. K. Ahn et al. [E391a Collaboration], hep-ex/0607016.

[11] A. Alavi-Harati et al. [KTeV Collaboration], Phys. Rev. Lett. 93, 021805 (2004) hep-ex/0309072. 
[12] A. Alavi-Harati et al. [KTeV Collaboration], Phys. Rev. Lett. 84, 5279 (2000) hep-ex/0001006.

[13] R. Barbieri and A. Strumia, hep-ph/0007265.

[14] M. Bona et al. [UTfit Collaboration], 0707.0636 [hep-ph].

[15] A. J. Buras et al., Phys. Lett. B 500 (2001) 161 hep-ph/0007085.

[16] M. Blanke et al., JHEP 0610 (2006) 003 hep-ph/0604057.

[17] G. D’Ambrosio et al., Nucl. Phys. B 645 (2002) 155 hep-ph/0207036.

[18] A. J. Buras, hep-ph/0109197.

[19] N. Arkani-Hamed et al., JHEP 0207 (2002) 034 hep-ph/0206021.

[20] T. Appelquist, H. C. Cheng and B. A. Dobrescu, Phys. Rev. D 64 (2001) 035002 hep-ph/0012100.

[21] P. Fayet, Phys. Lett. B 64 (1976) 159; Phys. Lett. B 69 (1977) 489; Phys. Lett. B 84 (1979) 416. G. R. Farrar and P. Fayet, Phys. Lett. B 76 (1978) 575.

[22] W. Altmannshofer, A. J. Buras and D. Guadagnoli, hep-ph/0703200.

[23] H. C. Cheng and I. Low, JHEP 0309 (2003) 051 hep-ph/0308199; JHEP 0408 (2004) 061 hep-ph/0405243.

[24] P. H. Frampton, Phys. Rev. Lett. 69 (1992) 2889. F. Pisano and V. Pleitez, Phys. Rev. D 46 (1992) 410 hep-ph/9206242. R. Foot et al., Phys. Rev. D 47 (1993) 4158 hep-ph/9207264.

[25] C. Bobeth et al., Nucl. Phys. B 726 (2005) 252 hep-ph/0505110.

[26] U. Haisch and A. Weiler, 0706.2054 [hep-ph].

[27] A. J. Buras and R. Fleischer, Phys. Rev. D 64 (2001) 115010 hep-ph/0104238.

[28] A. J. Buras et al., JHEP 0611 (2006) 062 hep-ph/0607189.

[29] A. J. Buras, M. Spranger and A. Weiler, Nucl. Phys. B 660 (2003) 225 hep-ph/0212143.

[30] M. Misiak et al., Phys. Rev. Lett. 98 (2007) 022002 hep-ph/0609232.

[31] U. Haisch and A. Weiler, hep-ph/0703064.

[32] G. Isidori et al., JHEP 0608 (2006) 064 hep-ph/0604074.

[33] G. Isidori and P. Paradisi, Phys. Rev. D 73 (2006) 055017 hep-ph/0601094.

[34] Y. Nir and M. P. Worah, Phys. Lett. B 423 (1998) 319 hep-ph/9711215.

[35] A. J. Buras, A. Romanino and L. Silvestrini, Nucl. Phys. B 520 (1998) 3 hep-ph/9712398.

[36] G. Colangelo and G. Isidori, JHEP 9809 (1998) 009 hep-ph/9808487.

[37] A. J. Buras et al., Nucl. Phys. B 714 (2005) 103 hep-ph/0408142.

[38] A. J. Buras et al., Nucl. Phys. B 566 (2000) 3 hep-ph/9908371.

[39] C. Smith, these proceedings.

[40] M. Blanke et al., JHEP 0701 (2007) 066 hep-ph/0610298.

[41] M. Blanke et al., JHEP 0612 (2006) 003 hep-ph/0605214.

M. Blanke et al., Phys. Lett. B 646 (2007) 253 hep-ph/0609284.

[42] Y. Grossman and Y. Nir, Phys. Lett. B 398 (1997) 163 hep-ph/9701313. 
[43] M. Blanke et al., 0704.3329 [hep-ph].

[44] A. Alavi-Harati et al. [KTeV Collaboration], Phys. Rev. D 67 (2003) 012005 [Erratum-ibid. D 70 (2004) 079904] hep-ex/0208007. J. R. Batley et al. [NA48 Collaboration], Phys. Lett. B 544 (2002) 97 hep-ex/0208009.

[45] A. J. Buras and L. Silvestrini, Nucl. Phys. B 546 (1999) 299 hep-ph/9811471.

[46] C. Promberger, S. Schatt and F. Schwab, hep-ph/0702169. 